\title{
Visualizing Decision Trees in Games to Support Children's Analytic Reasoning: Any Negative Effects on Gameplay?
}

\author{
Robert Haworth, Sousan Sheida Tagh Bostani, and Kamran Sedig \\ Cognitive Engineering Laboratory, Middlesex College, The University of Western Ontario, London, ON, Canada N6A 5B7 \\ Correspondence should be addressed to Kamran Sedig, sedig@uwo.ca
}

Received 22 October 2009; Revised 7 April 2010; Accepted 13 May 2010

Academic Editor: Ali Arya

Copyright ( $) 2010$ Robert Haworth et al. This is an open access article distributed under the Creative Commons Attribution License, which permits unrestricted use, distribution, and reproduction in any medium, provided the original work is properly cited.

\begin{abstract}
The popularity and usage of digital games has increased in recent years, bringing further attention to their design. Some digital games require a significant use of higher order thought processes, such as problem solving and reflective and analytical thinking. Through the use of appropriate and interactive representations, these thought processes could be supported. A visualization of the game's internal structure is an example of this. However, it is unknown whether including these extra representations will have a negative effect on gameplay. To investigate this issue, a digital maze-like game was designed with its underlying structure represented as a decision tree. A qualitative, exploratory study with children was performed to examine whether the tree supported their thought processes and what effects, if any, the tree had on gameplay. This paper reports the findings of this research and discusses the implications for the design of games in general.
\end{abstract}

\section{Introduction}

Digital games have become a significant force, not only in popular culture but also in the commercial market place. Popular games can sell millions of copies each, with revenue from the digital game industry reaching billions of dollars $[1,2]$. However, there is concern about the impact digital games may have on players, particularly on children $[3,4]$. Although only a quarter of all those who play digital games in the United States are children [2], they still spend an average of nine hours of their leisure time per week playing digital games [3].

Several studies have indicated that digital games can positively or negatively affect both physical as well as mental capabilities of players [4-6]. Currently, there is great hope that games can play an important role in promoting the highly-valued skills of the 21st century, namely, expert problem solving and complex communication [7]. In this paper we are interested in the design of digital games that promote mindful, analytical reasoning skills. Previous research indicates that strategies can be incorporated in digital games that support players' higher-order thought processes, such as analytical thinking and reasoning [8]. One design strategy that may affect players' reasoning in a digital game is incorporating visual structures in the game through which play is mediated $[8,9]$. Designing a game in this manner may change how players approach it, and thus may actually impact the resulting gameplay. If we are going to design digital games that prepare the next generation of players who are critical thinkers, one of the research questions that need to be explored is what effect interactive visual support structures would have on gameplay. For instance, if a game were designed such that gameplay would be mediated through visually explicit decision-support structures, how would the design affect gameplay and children's reasoning and decision-making strategies?

The purpose of this paper is to examine the above question. To do this, a game was designed and a study was conducted to investigate four subordinate, yet, inter related questions: (1) would the inclusion of the decision tree have any effect on children's reasoning and, consequently, their decision-making strategies? (2) would the inclusion of the tree have a negative effect on the quality of the gamethat is, its gameplay? (3) would mediating action through 
the tree have a negative effect on gameplay? and, (4) how can decision-support structures be incorporated in a game to promote better reasoning without negatively affecting gameplay?

The rest of this paper is organized as follows. The next section provides a brief background to the research. Then, a study that explored the above questions is described along with its results. The article ends with some conclusions and implications for other designers.

\section{Background}

To examine digital games in more detail, particularly with regard to their design, it is best to define the games that this paper examines. A variety of terms have been used such as computer games, video games, console games, and electronic games. We will use the term digital games to refer to all games that exist in a digital format, regardless of hardware platform, as opposed to games existing in a physical or non digital format. A wide variety of digital games exist, with one category being games that require analytical thinking and reasoning in order to play them effectively. Players of these games will analyze the state of the game, determine what decisions can be made, and then make the decisions that they deem are best for the current situation. These decisions could involve what paths to take through the game space, how to manipulate specific game objects, which objects to manipulate, and so on. The complexity of the game state and the decisions to make are dependent on the complexity of the digital game and are widely varied. For example, the decisions in a block-arranging puzzle game are less complex than those in a simulation game. This category of games is not synonymous with any genre of digital games, but instead contains games from multiple different genres.

The design of digital games often places gameplay as a core concept of design decisions $[10,11]$. This is because gameplay has a significant impact on the enjoyment of the game for players. Thus, the designer must ensure that gameplay is good for players to actually enjoy the game. Gameplay has a variety of definitions, ranging from the game's mechanics to the actual experience players feel while playing the game [11, 12]. Aarseth [13] has defined it as the actions, motives, strategies, and knowledge of players. We will consider gameplay in a similar manner, by defining it as the choices players make and the consequent actions they perform that leads to their overall enjoyment of the game. Another core concept of digital games is their structure [10, 14]. The structure of a game provides its formal definition, including its rules and logic, identifying what actions players can perform and how the game should respond. Not only does this structure describe the information needed to implement the game but also it directly influences how players can play the game. Gameplay contains the choices players make, but the choices available to players are determined by the structure of a game. In addition to defining the actions available to players, the structure also defines how players perform those actions. This allows the structure to better mediate the actions of the player through the interface of the game.
The interface of a game is comprised of two components: representation and interaction. Players of a digital game interact with the representations of the game's interface, which results in gameplay. In the context of digital applications, representations refer to collections of symbols that encode causal, functional, structural, and semantic properties and relationships of a represented world, either abstract or concrete, and interactions refer to players acting upon these representations and the representations reacting to these actions (for a similar conceptualization of this in the context of other interactive tools, see [15]). In digital games, the represented world is the internal information defined by the game structure. For the digital games this paper examines, the internal information is the choices or paths available to players. As part of playing a game, players will need to decide what actions to perform next and how to perform the desired actions. Most games are currently designed in such a way that the representations of the interface minimize the difficulty players have with performing their actions [16]. This is unrelated to the difficulty of the game. Designing games in this manner suggests that the thinking of players is directed towards the game world; the game does not support the reasoning required of players to decide on their next action, only on how to perform that action. However, it may be possible to change the focus of thinking for players on what actions to perform, rather than how, through the use of different representation and interaction strategies. One strategy with the potential to focus and aid the decisionmaking and reasoning of players is that of visual support structures $[8,9,17]$.

One type of visual support structure is a decision tree. This is a simple visual representation of all possible choices available to players and the consequences of each choice. Nodes of the tree encode choices or consequences, while the branches of the tree encode the act of making a choice and can be annotated with the specific option chosen or probabilities of that option being the correct one. Thus, the hierarchical structure of the tree encodes how the various choices are connected to one another, clearly indicating the consequences that arise from following a path from the root, or starting, node to any other node. When it encodes the internal information of a game, a decision tree provides an overview of all the possible choices for players as well as specific details on the consequences of any particular choice. Although decision trees are a part of decision-making strategies in artificial intelligence [18], they are also inherent in many types of games. For example, the flow of the storyline in games can be represented using a decision tree, with the nodes encoding the choices players can make at key plot points. Although these choices can simply change how the story unfolds, they can also affect whether players successfully complete the game (for an example of this used in training, see [19]). In other cases, such as strategy games, players must examine a variety of choices to determine the best order in which to perform them. For example, many strategy games involve players guiding a small military force to complete some goal. Players must determine how to arrange their forces, either for a better offensive position, defensive position, or to have a balance of both. Also, players 
often have to perform resource management. This involves allocating resources towards acquiring new forces, improving their existing forces, improving their overall infrastructure to acquire better resources, and so on. Each action players choose requires time to complete, and this will prevent players from performing other actions. Likewise, in many puzzle games the order in which players perform their actions is very important. Solving one part of the puzzle may prevent players from solving the rest of it, and thus players must examine their actions in a hierarchical manner; choosing to perform one set of actions may prevent them from choosing other required actions. For a visual structure to support the thinking of players, it should aid their reasoning process in some manner. To successfully complete many of the games this paper is interested in, players must understand the choices available to them and the consequences of those choices. As decision trees describe the choices and consequences in a game, a decision tree of the internal decisions of a game made explicit for players may act as a visual support structure to aid their decision-making process.

\section{Research Methodology}

A typical maze-like digital game was designed. The game required players to find their way from the starting point to the exit point of each of the game's maze-like puzzles, collecting a set of specific objects along the way. The challenge of the game revolved around finding the shortest path through each puzzle, which required players to plan their actions ahead of time to ensure no incorrect or unnecessary actions are performed. To compare differences among different support structures, four versions of this game were designed. Three of the versions included a decision tree and varied in how the players' actions were mediated. The other version did not include a decision tree. Broken down into a series of steps, the tree provided players with possible paths through the game's maze-like environment to help them understand how their choices were integrated into the overall solution for each puzzle. An exploratory study with children was conducted to investigate whether the inclusion of the decision tree would affect the children's reasoning and decision-making strategies as well as their enjoyment of the game. Additionally, the study would help us gain a better understanding of how to include a decision tree in such games.

3.1. Structure of the Game. To design the digital game used in the study, a typical activity was chosen. This activity involved searching for a path through a maze. A maze is a space composed of interconnected passageways that, at some point, form an uninterrupted path from a single starting point to a single finishing point. This suggests that a maze can be represented as a graph, with connections between nodes encoding individual passageways. Figures $1(\mathrm{a})$ and $1(\mathrm{~b})$ show a comparison between a simple maze and its graph.

The design of the digital game was based on a generalization of the chosen activity, and thus it did not contain a maze but a maze-like environment. This environment has the same structure as a maze-a graph-except its

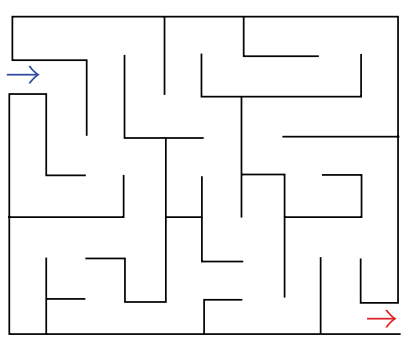

(a)

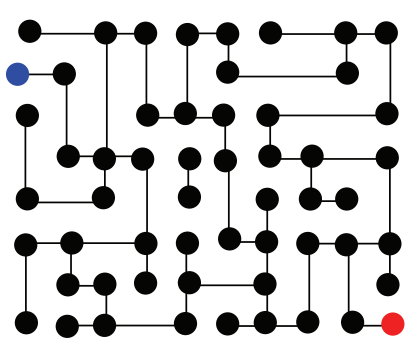

(b)
FIGURE 1: Comparison of a simple maze, represented traditionally (a) and as a graph (b). In both cases, the start is colored blue and the finish is colored red.

passageways are implicit. The goal of the game is to find a path from the start to the finish, performed by finding the connections between nodes of the graph. Thus, the decisions players must make while playing this game involve finding the connections and following the resulting path. At each node in the graph, players must decide which direction to follow in order to reach the next node. Some directions may not lead to anything, such as walls in a typical maze. The maze-like environment in the game is 2-dimensional, restricting movement to four directions: up, down, left, and right.

The digital game could include a graph as a visual support structure to aid players' reasoning. However, the interface of the game already includes representations that encode the internal structure as a graph, except in a more implicit manner as shown in Figure 2. The graph structure provides information about how various nodes are spatially related to each other. Players can trace a path from one node to another on a graph, but they can just as easily trace a path through game components. Additionally, the key decisions players must make in the game are about how to reach specific nodes in the fewest number of moves. Although players can determine this information by tracing paths through the graph, it does not explicitly provide the information that the players need. Thus, a graph will not provide the support needed. However, the information represented by a graph can also be represented as a tree. In this case, the game's internal graph can be represented as a decision tree. Each decision available to players-the directions available to move in-is encoded as a connection in the tree between a parent and its child nodes. As well, the result of each decision is encoded as child nodes in the tree. Directions that do not lead to a node in the graph are still encoded in the tree, albeit differently to indicate that there is no destination node. Since the graph contains cycles, the tree will contain duplicate nodes and duplicate paths. This will cause the tree to become infinite in size. This tree, unlike the graph, explicitly describes what paths are available from a given node to any other node and the sizes of these paths. This information is what players need in order to decide what direction to take to reach a desired node. The capability of the tree to provide players with all the possible decisions, and the results of those decisions, is what is expected of a visual support structure. 


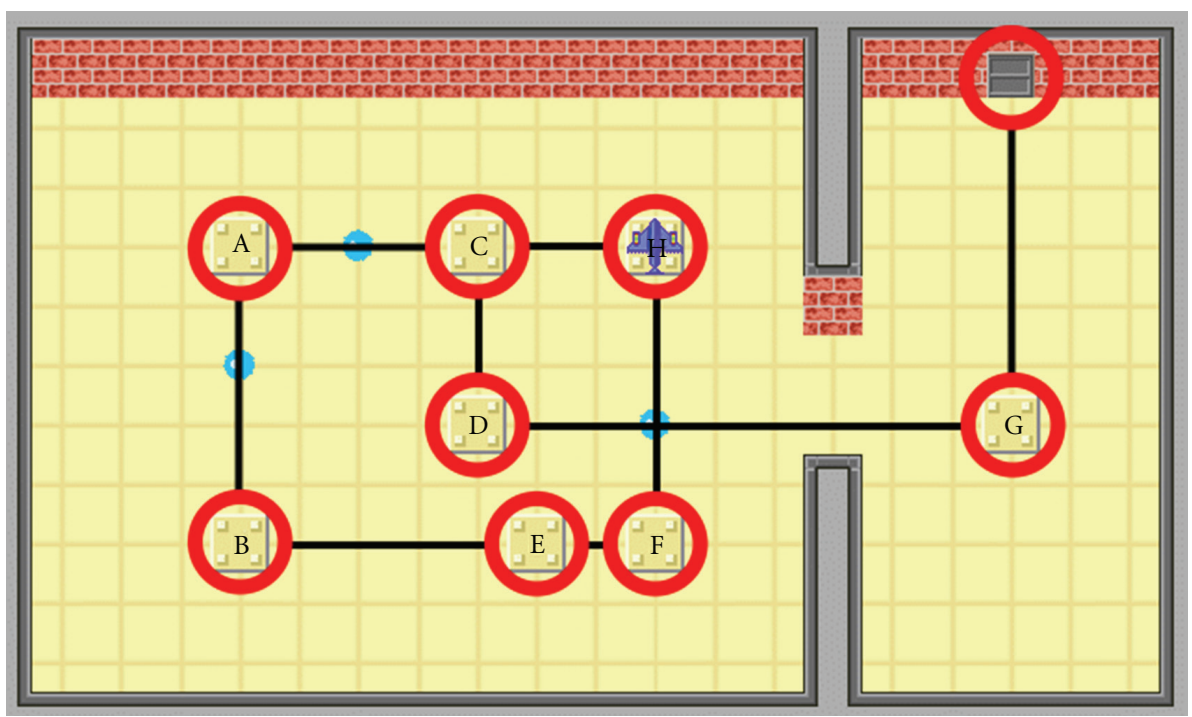

FIgURE 2: A typical puzzle in the game with the underlying graph structure superimposed over it. Connections to walls are not shown.

3.2. Studied Game. A digital maze-like game was designed and implemented for the study. The game is divided into multiple levels of increasing difficulty. The objective of each level is for players to find a path from their starting location to the exit. Before reaching the exit, players must collect all the colored balls on the level by moving through them. The game encodes the nodes of the underlying graph as flat platforms, as shown in Figure 2, and the players' representative character, their avatar, is encoded as a small airplane. Players move from one platform to the next without being able to control their movement between platforms. For example, when players choose to move to the right, their avatar begins moving to the right and will not stop until it reaches another platform.

Walls are placed around and within each level at specific locations. Colliding with a wall destroys the players' avatar, forcing them to restart the level. To complicate the path finding, other components such as arrows are placed in various locations in each level. These arrows change the direction of the avatar in a specific way. See Figure 3 for how these arrows relate to the underlying graph. The color of the avatar must match the color of the collectable balls as well. A spinning ring component changes the avatar to a specific color, depending on the ring. Figure 4 shows the main puzzle area of a typical level in the game containing labeled platforms, rings for color changing, the collectable balls, and other operators that change the players' direction.

The background to each level contains a grid pattern, with the intent of helping players identify the path their avatar will follow. As well, the components which redirect the avatar are encoded in as obvious a manner as possible, such that players can easily identify the effects of colliding with different components. For instance, an operator that moves the avatar to the left is encoded as an arrow pointing to the left.

The game rewards players for reaching the exit via the shortest route and penalizes them for using a route that is too long. Many of the levels are designed to trap players, forcing them to restart, if they are not careful about which path they choose. As well, players are penalized for colliding with a wall. However, there is no limit to how often a level can be repeated nor is there a time limit; players can take as long as they need to decide their next move. We assumed that this would encourage players to carefully examine each level in order to find the best path.

3.3. The Tree Diagram. The internal decision tree of the game is represented as a tree diagram. The destinations along any given path are encoded as circular nodes in this diagram, while the directions available to players are encoded as lines connecting those nodes. To ensure players understand which direction the line encodes, a small arrow is placed over the destination node. The nodes of the diagram are colored to indicate the color the players' avatar will become upon choosing that direction. If a collectable ball is along the path to a destination node, a small ball of the appropriate color is shown on the connecting line. To provide consistent mapping between the tree diagram and the puzzle area, each platform is labeled with a letter. The same label is used in the tree diagram to indicate which platform a specific path will lead to. The exit and walls are encoded on the diagram with the same symbols as those used in the main puzzle area. Operators that change direction are not included in the diagram.

Figure 5 shows the tree diagram for the puzzle area shown in Figure 4. As an example of reading this diagram: if players want to reach the exit from their starting node of $\mathrm{N}$, they must move down to node $\mathrm{A}$, down again to node $\mathrm{D}$, and then right towards the exit. Note that the tree provides the initial direction of movement but not possible redirections that may occur before reaching the destination. Players only need to know the initial direction that will take them to a destination node, as those are the directions they have to choose from. 


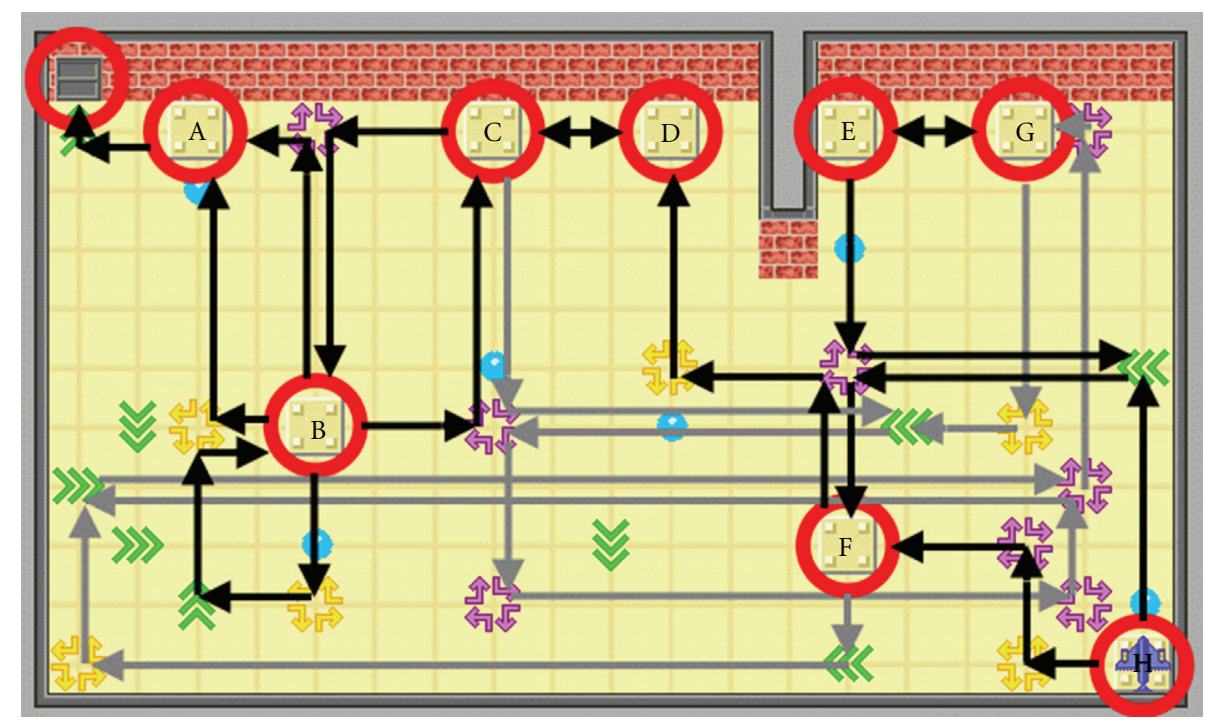

FIGURE 3: A puzzle that shows how redirection operators complicate the underlying graph structure. For example, note how the lighter series of arrows always lead to node G. Connections to walls are not shown.

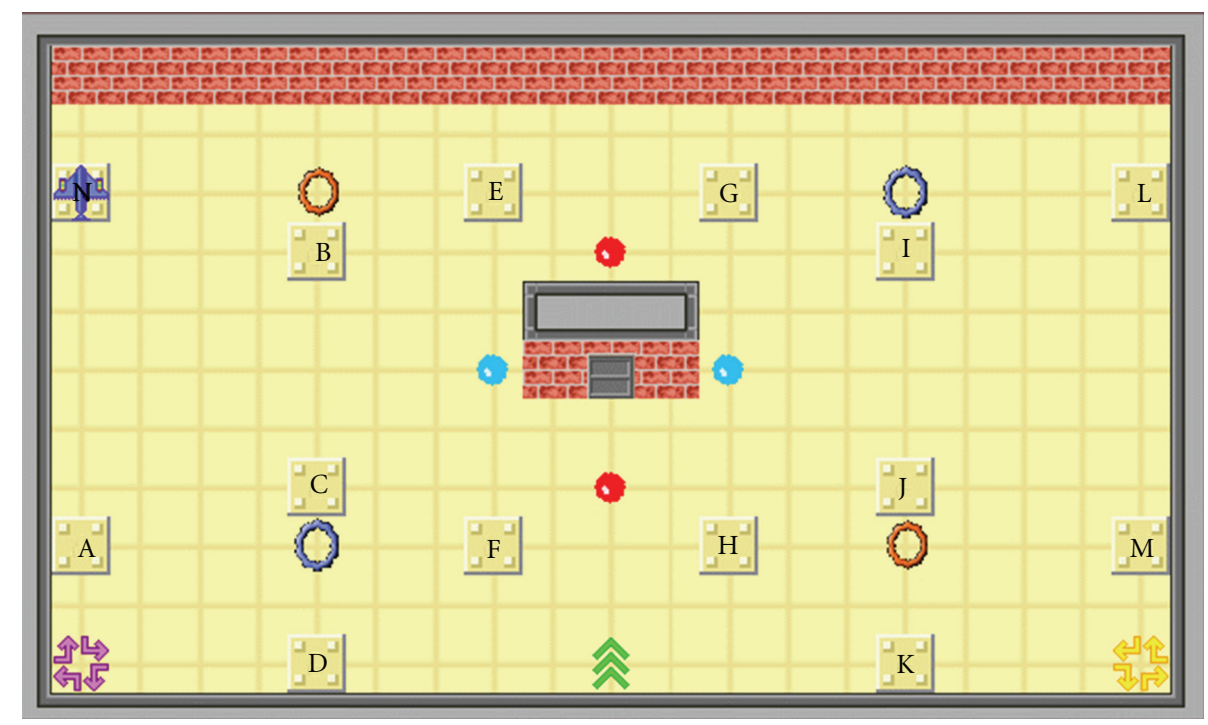

FIGURE 4: The main puzzle area of the game, with players starting at the $\mathrm{N}$ platform and needing to finish at the gray door in the middle.

The entire decision tree cannot be represented all at once, as it is infinite in size. Instead, the tree is represented only to a certain depth. The resulting diagram also shows multiple similar paths. Therefore, only nodes that reveal new information are encoded in the diagram. If the tree already encodes the path from one node to another, a dashed line is used as the encoding instead.

Although the tree diagram is not infinite in size, it is usually too large to be completely displayed on the screen. To overcome this, an additional side panel is added that includes a minimap of the tree diagram. A panning glass is superimposed over this minimap to highlight what section of the diagram is currently displayed, and players can move this panning glass with the mouse. This allows players to see the entire tree at a glance and to view a specific section of it in more detail. An example of the side panel is shown in Figure 6, containing a minimap for the diagram from Figure 5.

3.4. Game Variations. The four versions of the game each have the same rules and goal, as well as the same levels to complete. All versions contain the main puzzle area, shown in Figure 4, which displays the level and the players' avatar. Only three versions contain the tree diagram and the side panel, shown in Figures 5 and 6 respectively. Each version also differs in how players interact with the game.

3.4.1. Interactive Tree and No Keys $(I T+N K)$. In this version, both the decision tree and the puzzle are present. The tree is interactive and players' actions are solely mediated through 


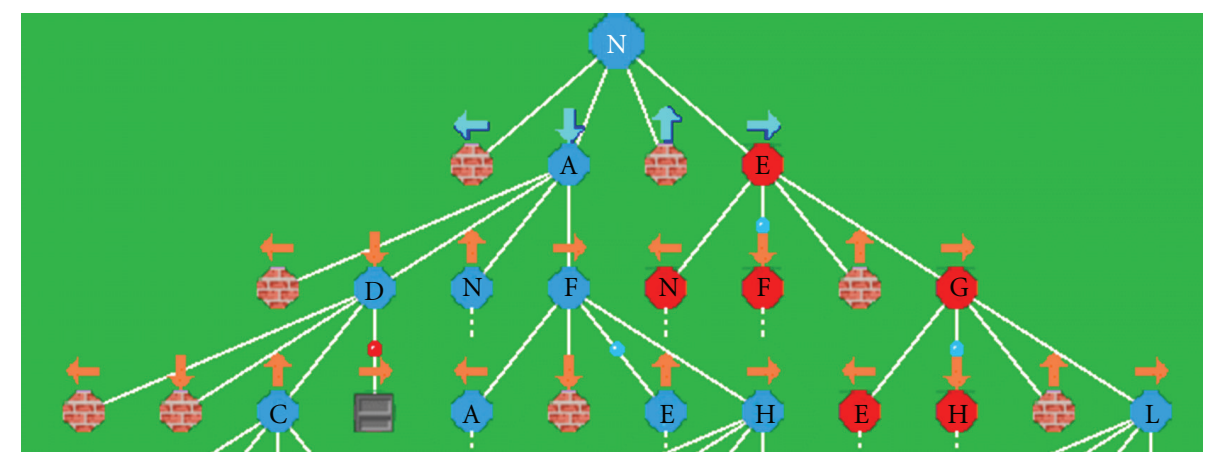

FIgure 5: The tree diagram for the puzzle area in Figure 4.

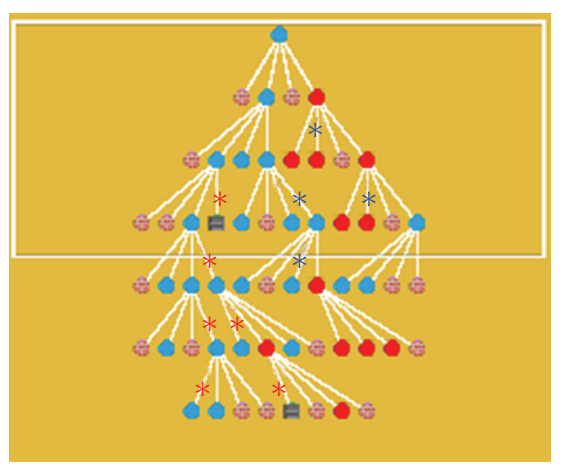

FIGURE 6: The side panel showing the complete tree diagram from Figure 5 .

the tree. However, the keys are inactive and players cannot express their choices through the keyboard. To express their choice of action, players click on the corresponding arrow directly below the root node of the tree diagram. For example, if players have the tree diagram shown in Figure 5 and want to move from node $\mathrm{N}$ to node $\mathrm{A}$, they would click on the blue arrow just above node A that points down. These interactive arrows are colored differently, to indicate this functionality is available, but all the other arrows are inactive and cannot be clicked on. Given that the tree mediates players' actions, it is assumed players would also use it to reason about their decisions. Refer to Figure 7 for a screenshot of this version.

3.4.2. Noninteractive Tree and Keys $(N I T+K)$. In this version, both the decision tree and the puzzle are present. The keys are interactive and players' actions are solely mediated through the keyboard. However, the tree is inactive and players cannot express their choices through the tree. To express their choice of action, players press the corresponding arrow key on the keyboard. As the tree only exists as a visual support structure, and does not mediate any actions, it is assumed players would not use it as strongly to reason about their decisions. Refer to Figure 7 for a screenshot of this version.

3.4.3. Interactive Tree and Keys $(I T+K)$. In this version, both the decision tree and the puzzle are present. Both the keys and the tree are interactive and players' actions can be mediated through either of these. Players express their choice of action in the same manner as either version IT+NK or $\mathrm{NIT}+\mathrm{K}$. That is, players can click on the corresponding arrow in the tree or press the corresponding arrow key on the keyboard. This version reveals the preference players have over how to choose their actions, in addition to the same information as versions IT+NK and NIT $+\mathrm{K}$ depending on how players interacted with the game. Refer to Figure 7 for a screenshot of this version.

3.4.4. Keys $(K)$. In this version, only the puzzle is present. Players' actions are mediated solely through the keyboard and there is no visual decision-support structure in the form of the tree. Players express their choice of action in the same manner as version NIT $+\mathrm{K}$ : by pressing the corresponding arrow key on the keyboard. This version is thus similar to typical digital games that do not have visual structures to support the decision-making processes of players. This version is used to compare how the inclusion of the decision tree affected gameplay and reasoning, as the tree is not available in it. Refer to Figure 8 for a screenshot of this version.

3.5. Exploratory Study. An exploratory study was conducted to compare the four versions of the game. The purpose of this study was to examine whether the inclusion of a decision tree in the game would have any effect on the players' analytical reasoning and decision-making strategies and, if so, how. More importantly, the study also examined how including the decision tree, independent of its other effects on the participants, affected the gameplay of the digital game, or the participants' enjoyment of the game. As this was only an exploratory study, the primary objective was to look for the existence of such effects to determine future areas of research. Therefore, we gathered qualitative data about how the participants played the game, the manner in which they utilized the decision tree, and why they did or did not enjoy playing the game. The degree to which the support structure affected the gameplay and the players' reasoning was not tested.

A total of 13 children, 7 boys and 6 girls, participated in this study. Their ages ranged from 9 to 13 years old. As this was a qualitative study, the sample size did not need to be as 


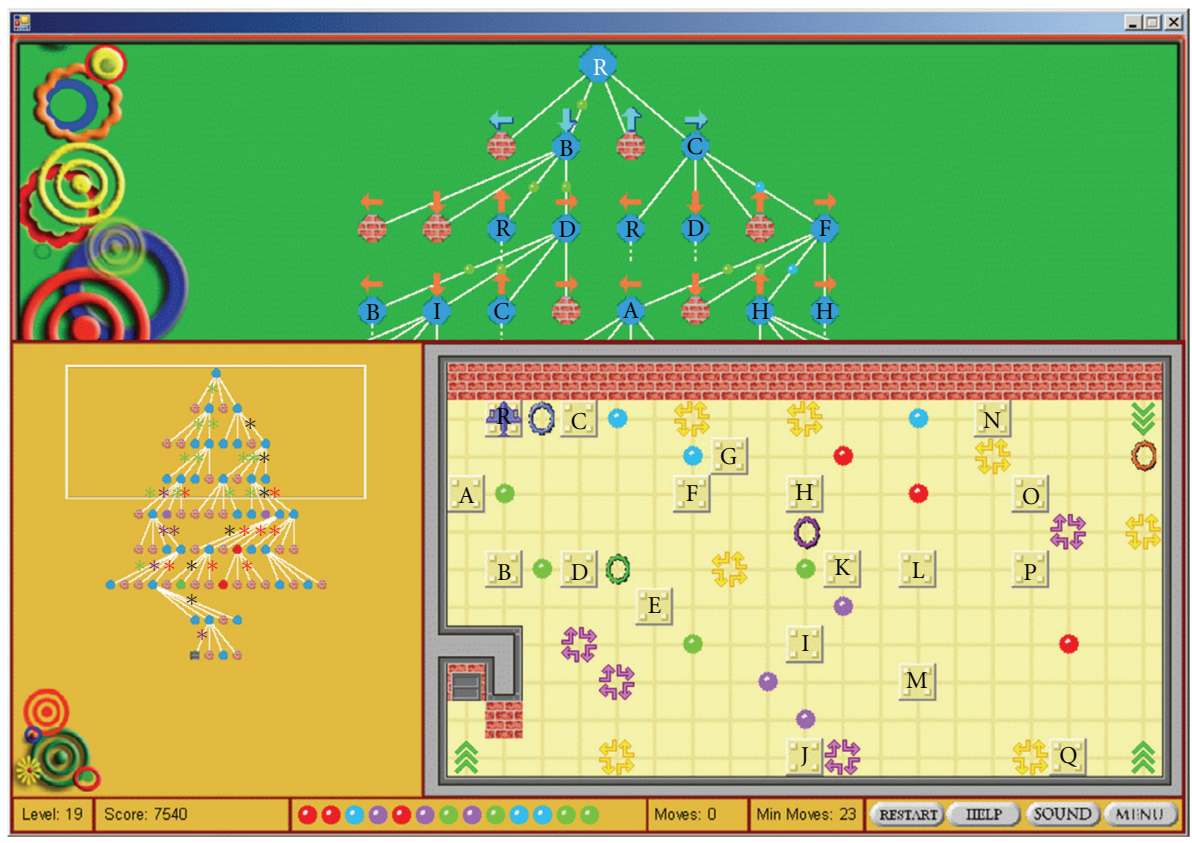

Figure 7: Versions IT + NK, NIT $+K$, and $I T+K$ of the game, all of which contain the tree diagram.

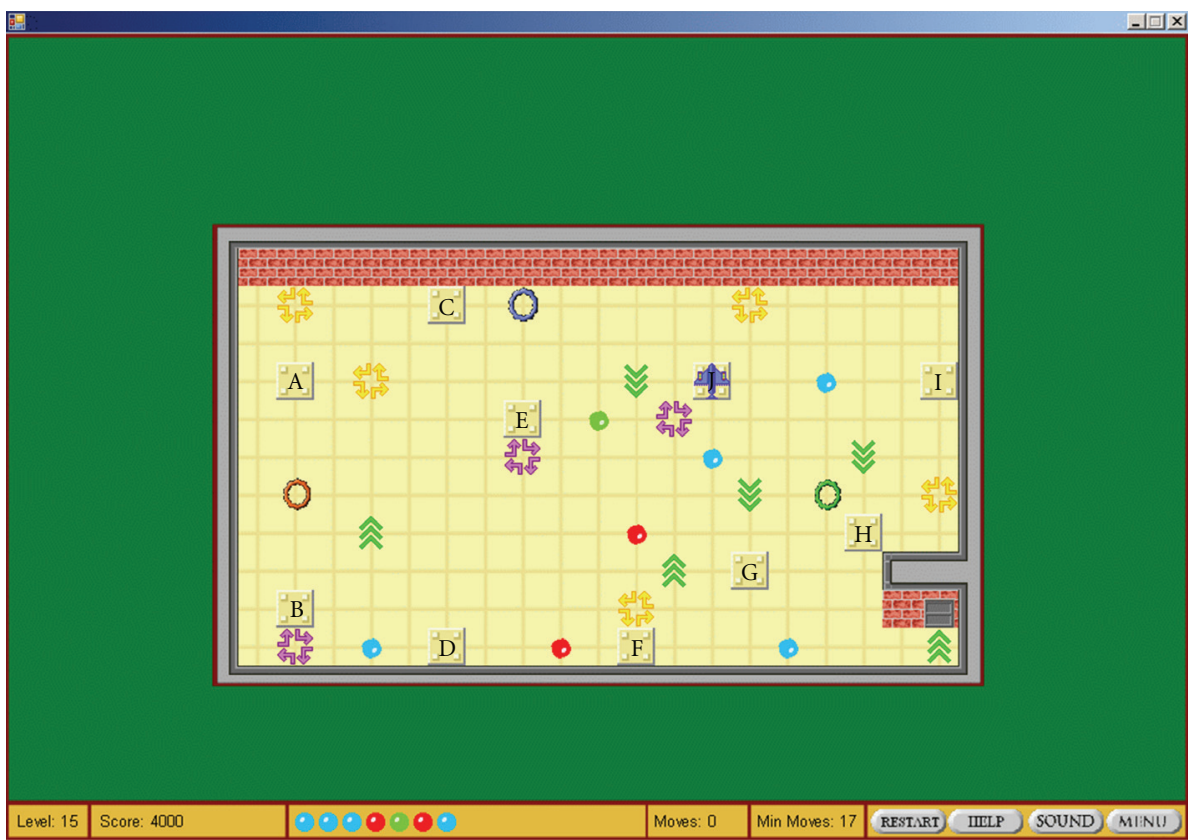

Figure 8: Version K of the game, which does not contain the tree diagram.

large as required for a quantitative study [20]. None of the participants had played the digital game before. Even though some of them had seen factor trees in their mathematics classes, they had not been exposed to abstract decision trees as represented in this game. The participants were computer literate and had previously played other digital games, with varying degrees of regularity.

Three sources of data were used to perform the analysis and evaluation of the digital game: (1) video transcripts, obtained from video recordings of the participants' interactions with the game; (2) interview transcripts, obtained from video recordings of post hoc interviews with the participants; and (3) direct observations, obtained from the recordings made of participants' overall patterns of usage and body language not easily captured through video recordings.

The participants were randomly distributed into four groups, with each group focused on one version of the game. Each participant was paired with another participant or, in 
the absence of other participants, with one of the researchers so that the participant could think aloud more naturally. This pairing was based on the codiscovery learning method, encouraging the participants to converse with each other in a more natural way [21]. This also provided the researchers with a better understanding of the participants' thought processes when interacting with the game. The participants were given a brief introduction to the game and a brief period of time to familiarize themselves with it. After that, the participants were asked to play through the entire game. Each of the play sessions were approximately 90 minutes in length, composed of two consecutive 45-minute sessions separated by a 10 -to-15 minute break. The groups were videotaped for the entire play session, with the intent of capturing body language and discussions between the participants. This data would provide insight on the decision-making strategies players used to decide on their actions, how they performed their actions, their interpretation of the outcome of their actions, how much planning they were performing, and so on. Researchers also asked questions at various points to encourage the participants to clarify or expand on the reasoning used in their decision-making strategies and on any difficulties they were having with completing the game. All the groups were asked questions of this nature, but the questions were asked sparingly and not in an attempt to distract the participants from the game. Answers to these questions were recorded using both video and written notes. After the completion of the game, the participants were interviewed and asked a variety of open-ended questions regarding their interaction with the game. These questions were meant to understand the participants' overall thoughts and feelings about the game, what they did or did not enjoy about the game and why, why they played the game in the manner they did, whether they thought they used the decision tree at all, what they would change to improve the game, and other similar questions. Analysis of these questions would aid in understanding whether the decision tree had any effect on their decision-making strategies and gameplay. After all participants were interviewed, they were shown the other three versions of the game. They played the most challenging levels and were asked to express their thoughts and feelings about the other versions, and how the other versions compared to the one they originally used. All groups were shown the versions in the same order, excluding the version they originally played, starting with $\mathrm{IT}+\mathrm{NK}$, then $\mathrm{NIT}+\mathrm{K}, \mathrm{IT}+\mathrm{K}$, and finally $\mathrm{K}$.

\section{Results}

This section provides the results of the study. This includes comments made by the participants during their interaction with the game and during the interview, as well as notes taken by the researchers.

4.1. Version $I T+N K$. The participants approached this version primarily with a trial-and-error strategy, usually only planning one or two moves ahead. To find a path from their current location to a desired location the participants manually traced the connections between platforms in the puzzle area. After finding the path they wanted, they would click the arrow to move in that direction without examining the choices provided by the decision tree. There were several occasions where one pair of participants chose a direction in the tree even though it told them that their choice led to a wall.

However, after progressing halfway through the game the participants noticed the paths were quite difficult to find in the puzzle area. Some of them started examining the tree to look for potential routes, but it was still only one or two moves ahead. The participants rarely used the side panel, and typically examined the first three rows that were displayed instead. Once the participants reached the levels near the end of the game, the tree was more commonly used to check their current state: to find which directions would lead to a wall, which paths would lead back to their current location, and which paths would lead them out of a trap.

The participants did not like this version, especially compared to the NIT $+\mathrm{K}$ and $\mathrm{IT}+\mathrm{K}$ versions. The main complaint was the use of the mouse to click on the arrows for movement, as shown by comments such as

"I think it would take much longer to play this version. We are not used to clicking as much as we are used to using the arrow keys."

and

"I still think the arrow keys are better to play. Clicking is kind of hard to play but some people may like it better."

The participants did not complain about the tree diagram though and agreed that the game was fun:

"Yes, that was fun because it was challenging."

4.2. Version NIT $+K$. Participants approached this version much the same as other participants initially approached version IT+NK. They would trace their paths manually and would choose a direction that led them towards the next closest collectable ball. As in version IT+NK, manually tracing a path became more difficult as the participants progressed through the game.

However, the participants who played this version did not examine the tree as readily. When they did start to examine the tree, they found it difficult to use:

\section{"At first I didn't know what it was, but when I understood it, it was really helpful."}

It took these participants longer to effectively use the tree, but they used it quite extensively by the later levels. The tree was used for the same purposes as in version IT+NK: examining their current state to find a way out of a trap or to locate areas to avoid. When asked about the tree, one participant said:

\footnotetext{
"We used the tree because it was easier compared to following all those arrows."
} 
This version was preferred over version IT+NK because of the mode of interaction:

"I like this version because using the arrow keys makes it more fun."

4.3. Version $I T+K$. Although this version allowed participants to interact with the game through the tree diagram or using the keyboard, all the participants chose to use the keyboard. These participants approached the game much the same as those who used version NIT $+\mathrm{K}$, both initially and after experimenting with the tree diagram.

Many of the participants stated that this version was the best due to the choice of how to interact with the game:

"I think this version would be even better. Pressing the tree when you need it is a good idea."

and

"I like this version even better. If somebody likes one of them [i.e.,: arrow keys or clicking], it won't be hard for them to play because they can use one or the other."

4.4. Version $K$. This version did not have the tree diagram, so participants played it as they would a typical maze-like game. As in the other versions, participants manually traced a path to find the next desired platform and then moved in the appropriate direction. These participants even completed the easier levels faster than the participants of the other versions. However, once the participants reached the harder levels they found it very difficult to trace their paths. These participants often used extensive experimentation to figure out all the available routes before they managed to find the correct path.

When the participants who played version $\mathrm{K}$ were shown the other versions their responses were mixed. One said:

"I like this version because it tells you your options and it's easier. I think it is less challenging."

while the other disagreed

"Actually I think it is a little bit harder, because you have to concentrate on the tree instead of the puzzle."

The participants who initially played the other versions did not like version $\mathrm{K}$. They were used to playing the game with the tree diagram and felt the game was worse when it was not available:

"I think this might be a bit more confusing."

"I still prefer the version that I played [IT $+\mathrm{K}]$. Having the tree up there is kind of better because when you are stuck it really helps."

"This one looks much harder. There is nothing to tell you these are the possibilities and you are in danger. In this version $[\mathrm{K}]$ you just have to keep doing it to see what will happen, but in the other versions you can actually see this leads there. We used the tree to see how to go to somewhere in the hard situations."

\section{Discussion}

As there was no enforced time limit, participants could take their time playing the game and had the opportunity to consider their actions before choosing what to do. However, the participants that were used to playing faster-paced games approached this game as though they had to act immediately. They did not take the time to examine their choices in detail and were not able to plan their actions far enough in advance to avoid the various traps. The other participants took their time and tried to plan out their moves, though initially it was not very far ahead. All the participants, regardless of which version they used, had difficulty with the harder levels. This was particularly true of the last three levels of the game. Although the participants with access to the decision tree did not initially use it, all of them used it to assist them in the harder levels.

At first, the tree was used to simply rule out which directions would lead the participants into a wall, automatically removing undesired outcomes. Then, they examined which paths would lead them into traps, at first only noticing obvious traps and then noticing some more subtle ones. By the more difficult levels, some participants were even using the tree to determine how to reach certain desired areas. For example: they needed to be a specific color, or needed to collect a specific ball, and would look through the tree to find the appropriate path. The participants who did not use the tree played through the entire game using luck and experimentation to complete the levels, and found it quite difficult to choose which directions would benefit them in the long run. This suggests the inclusion of the decision tree may have positively affected the participants' reasoning skills, in that it focused their reasoning on how to complete the overall level rather than on how certain platforms were connected. It may also have affected their decision-making strategies by favouring directions that would help them complete the game rather than simply reach the next platform. While this may be the case for the groups that extensively used the tree, how much of an impact the decision tree had on the participants' reasoning requires further investigation.

Only four of the participants were concerned about their score, and used this as an incentive to find the shortest path. When the path became too long, they would restart the level to get a shorter path but only if the level was not too complicated. The more complicated levels took too long for them, and they did not mind losing some score if it meant they would not have to redo the level. However, if they would lose a lot of score, they still restarted the level. The other participants were less concerned about their score, or considered their score irrelevant. Some liked to find the shortest route while others were only interested in completing each level. These participants only restarted when they were stuck, and would not redo the level to find a shorter path if they were close to the level's exit. Thus, the score awarded for finding the shortest path had minimal effect on the participants' motivation to play the game. Additionally, finding the shortest path did not have the highest priority for all of the participants, as many were content simply to finish the harder levels. This suggests 
something other than scoring may need to be used in the design of the game for future studies in order to encourage long-term planning through the maze. The length of the paths through the puzzles may also need to be adjusted, though this does not suggest that the puzzles were too difficult as discussed below.

How the participants enjoyed the game was determined by their reaction to it. The participants did not mind the inclusion of the decision tree, and many did not like version $\mathrm{K}$ because it did not include the tree. This suggests the inclusion of the decision tree, as a visual support structure, did not negatively affect the participants' conscious enjoyment of the game. However, the participants did not like the decision tree mediating their actions, as implemented in version IT+NK. As well, all the participants who played version $\mathrm{IT}+\mathrm{K}$ chose to interact with the game using the keyboard and not the mouse. The participants who played IT $+\mathrm{K}$ preferred the keys to mediate their actions and not the decision tree. This could be attributed to the decision tree not being integrated into the game well enough. Many of the participants found it difficult to click on the individual arrows, as they were smaller than the nodes of the tree. They also became frustrated at having to keep moving the mouse and clicking, suggesting that the arrow keys provided a more natural method of interaction. This suggests our implementation of mediating actions through the decision tree was inadequate and may need to be altered for future studies.

Most of the participants felt that the game would be too difficult without the existence of the decision tree. Some of the participants who played version $K$ struggled with the more difficult levels. This suggests that the challenge in the game was balanced towards the players utilizing the decision tree. This is significant because challenge is a key component for enjoyment in a game $[17,22]$. Although the inclusion of the decision tree was only helpful once the participants became adjusted to it, it was not a detriment to the game. As one participant said:

\begin{abstract}
"Trees are a turn off since we had seen enough trees in math class, but it doesn't really distract me from playing the game. It was helpful from time to time, so you don't have to look at all the ways to find a path."
\end{abstract}

\section{Conclusions}

This research examined whether the inclusion of visuallyexplicit decision-support structures in the design of a digital game, and mediating its gameplay through these structures, would affect its gameplay and the reasoning and decision-making strategies of its players. These effects were examined through an exploratory study with children, using a simple maze-like digital game. Four variations of the game were developed, of which one did not contain the support structure. The other three variations contained a visual support structure in the form of a decision tree, and mediated the actions of players through this tree or through the keyboard.
From the study, four tentative conclusions can be made. First, the inclusion of the decision tree may have positively affected the players' reasoning by aiding and focusing their decision-making process. Second, the inclusion of the decision tree did not have obvious negative effects on the players' enjoyment of the game. Third, our implementation of mediating actions through the decision tree seemed to have negatively affected the players' enjoyment of the game. And fourth, designers should be careful about how the actions of players are mediated in a digital game if decision-support structures are included to ensure that gameplay is not negatively affected. Although the study participants whose actions were mediated by the decision tree were able to utilize it better, they preferred having their actions mediated by the keyboard. This suggests a more natural implementation of mediating their actions was required and should be examined in future studies of this nature. The inclusion of the decision tree did decrease the study participants' perceived challenge of the game, but they felt the game would be too difficult if the tree was not included. Just as with any digital game, designers should examine all components of the game that affect gameplay to ensure that the difficulty is balanced. This includes any support structures incorporated into the game.

Although these conclusions require further research to verify, the ideas behind them still have implications for the general design of digital games. Incorporating a decision tree as a support structure may provide a benefit to the reasoning and decision-making strategies of players. However, designers must ensure that the decision tree clearly and accurately represents the choices that players need to make while playing the game. Otherwise, as suggested in this study, players may have difficulty understanding the support structure and may not receive its full benefits. Likewise, mediating the players' actions through a decision support structure must be designed such that the interaction is natural and does not detract from the enjoyment of the game.

Our investigation of the research question was conducted using an exploratory study. Additional, more rigorous studies need to be conducted in order to determine the validity of the findings of this research and whether these findings can be generalized. Further research in this area will also provide clarity on the full effects of visual support structures. For example, we have examined how to support directionalbased decisions for navigation, but what about games that require different decisions? How can designers support more complex decision-making, such as managing a large interconnected system? As well, supporting different decisionmaking processes may require visual representations that are different from a decision tree. What other visualizations can be used? Most importantly, how will designers be able to know which visualization is most beneficial to the decision-making process required for their digital game? These questions need to be investigated in order for designers to gain a better understanding of how to design enjoyable digital games that will promote the desired reasoning skills of tomorrow. 


\section{Acknowledgments}

This research has been funded by the Natural Sciences and Engineering Research Council of Canada. The authors would like to thank the children who participated in this study. The authors would also like to thank the reviewers for their comments and suggestions for improvement.

\section{References}

[1] S. Price, "What sells where and why?" Lecture Notes from Game Developers Conference, Europe, 2002.

[2] Entertainment Software Association (ESA), "Essential facts about the computer and video games industry," June 2009, http://www.theesa.com/facts/pdfs/ESA_EF_2009.pdf.

[3] D. A. Gentile, P. J. Lynch, J. R. Linder, and D. A. Walsh, "The effects of violent video game habits on adolescent hostility, aggressive behaviors, and school performance," Journal of Adolescence, vol. 27, no. 1, pp. 5-22, 2004.

[4] A. Lager and S. Bremberg, "Health effects of video and computer game playing - a systematic review," Report for the National Swedish Public Health Institute, 2005.

[5] B. G. Southwell and K. O. Doyle, "The good, the bad, or the ugly? A multilevel perspective on electronic game effects," American Behavioral Scientist, vol. 48, no. 4, pp. 391-401, 2004.

[6] C. D. Rodeheffer and C. P. Barlett, "A meta-analytic review of video/computer game play on short-term cognitive performance," in Proceedings of the Annual Meeting of the International Communication Association, Montreal, Canada, 2008, http://www.allacademic.com/meta/p230187_index.html.

[7] H. A. Spires, "21st century skills and serious games: preparing the N generation," in Serious Educational Games: From Theory to Practice, L. A. Annetta, Ed., pp. 13-23, Sense Publishing, Rotterdam, The Netherlands, 2008.

[8] K. Sedig, "From play to thoughtful learning: a design strategy to engage children with mathematical representations," Journal of Computers in Mathematics and Science Teaching, vol. 27, no. 1, pp. 65-101, 2008.

[9] E. N. Wiebe, "Data visualization and gaming," in Serious Educational Games: From Theory to Practice, L. A. Annetta, Ed., pp. 47-55, Sense Publishing, Rotterdam, The Netherlands, 2008.

[10] K. Salen and E. Zimmerman, Rules of Play: Game Design Fundamentals, MIT Press, Cambridge, Mass, USA, 2004.

[11] C. Fabricatore, "Gameplay and game mechanics design: a key to quality in videogames," in Proceedings of OECD-CERI Expert Meeting on Videogames and Education, Santiago de Chile, Chile, 2007.

[12] L. Ermi and F. Mäyrä, "Fundamental components of the gameplay experience: analysing immersion," in Proceedings of the DiGRA Conference, Vancouver, Canada, 2005.

[13] E. Aarseth, "Playing research: methodological approaches to game analysis," in Proceedings of the 5th International Digital Arts and Culture Conference, Melbourne, Australia, 2003.

[14] A. Rollings and E. Adams, Andrew Rollings and Ernest Adams On Game Design, New Riders Games, 2003.

[15] K. Sedig and M. Sumner, "Characterizing interaction with visual mathematical representations," International Journal of Computers for Mathematical Learning, vol. 11, no. 1, pp. 1-55, 2006.
[16] A. H. Jørgensen, "Marrying HCI/Usability and computer games: a preliminary look," in Proceedings of the 3rd Nordic Conference on Human-Computer Interaction, pp. 393-396, Tampere, Finland, 2004.

[17] K. Sedig, "Toward operationalization of 'flow' in mathematics learnware," Computers in Human Behavior, vol. 23, no. 4, pp. 2064-2092, 2007.

[18] S. J. Russell and P. Norvig, Artificial Intelligence: A Modern Approach, Prentice Hall, Upper Saddle River, NJ, USA, 2nd edition, 2002.

[19] A. Gordon, M. van Lent, M. van Velsen, P. Carpenter, and A. Jhala, "Branching storylines in virtual reality environments for leadership development," in Proceedings of the 16th Innovative Applications of Artificial Intelligence Conference (IAAI '04), pp. 844-851, July 2004.

[20] J. H. McMillan and S. Schumacher, Research in Education: A Conceptual Introduction, Allyn \& Bacon, 5th edition, 2000.

[21] S. Kennedy, "Using video in the BNR usability lab," in Readings in Human Computer Interaction: Towards the Year 2000, Morgan Kaufmann, San Francisco, Calif, USA, 2nd edition, 1995.

[22] R. Rouse, Game Design Theory and Practice, Wordware Publishing, Plano, Tex, USA, 2001. 

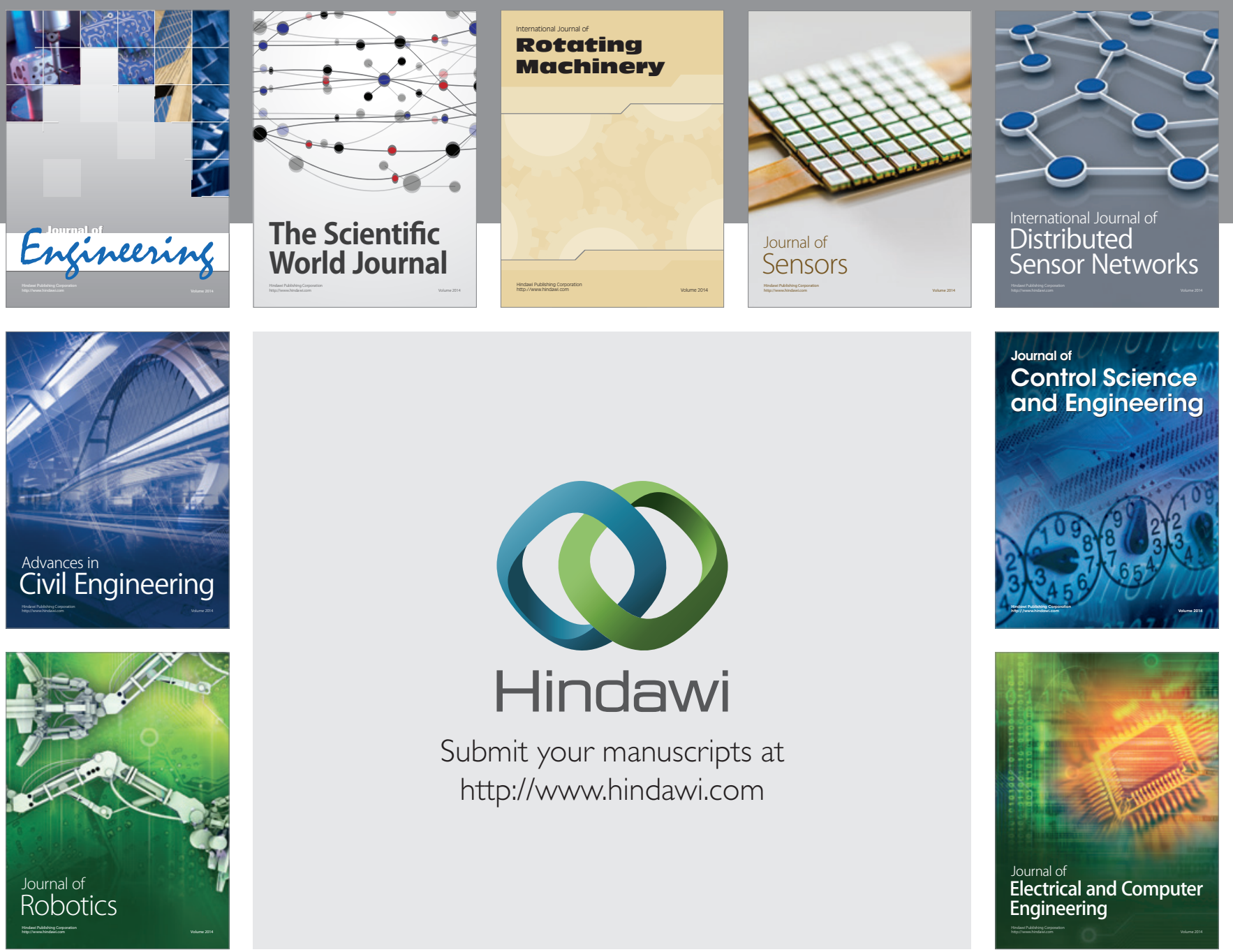

Submit your manuscripts at

http://www.hindawi.com
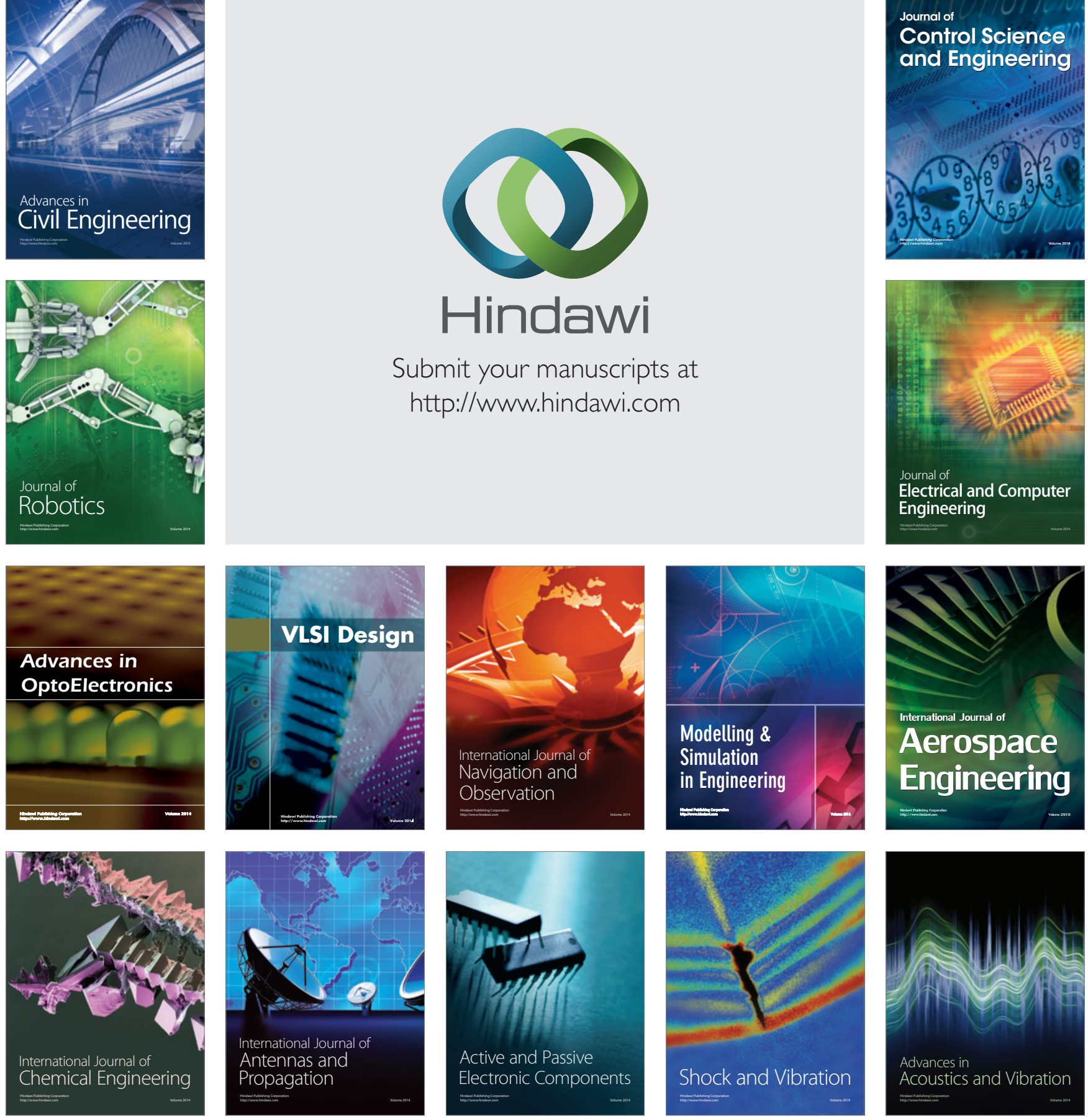